\title{
Stimulation of the Anterior Nucleus of the Thalamus for Epilepsy: A Canadian Experience
}

\author{
Manuel L. Herrera, Ana Suller-Marti, Andrew Parrent, \\ Keith MacDougall, Jorge G. Burneo (D)
}

\begin{abstract}
Objective: To describe the experience with Anterior Nucleus of the Thalamus-Deep Brain Stimulation (ANT-DBS) for the treatment of epilepsy at a Canadian Center. Methods: All patients who underwent ANT-DBS implantation between 2013 (first patient implanted at our center) and 2020 were included. These patients had therapy-resistant epilepsy (TRE), were not candidates for resective surgery, and failed vagus nerve stimulation (VNS) treatment. Baseline of monthly seizure frequency was calculated within 3 months prior to VNS placement. Monthly seizure frequency was assessed at different points along the timeline: 3 months before ANT-DBS implantation as well as 3, 6, 12, 24, 36, 48, 60, and 72 months after ANT-DBS device placement. At each time point, seizure frequency was compared to baseline. Results: Six patients were implanted with ANT-DBS. Three (50\%) patients had multifocal epilepsy, one (16.6\%) had focal epilepsy, and two (33.4\%) had combined generalized and focal epilepsy. Two patients with multifocal epilepsy experienced a seizure reduction $>50 \%$ in the long-term follow-up. Three (50\%) patients did not showed improvement: two with combined generalized and focal epilepsy and one with focal epilepsy. There were not surgical or device-related side effects. Two (33.3\%) patients presented mild and transient headaches as a stimulation-related side effect. Conclusion: ANT-DBS is an effective and safe treatment for focal TRE. Our experience suggests that patients with multifocal epilepsy due to regional lesion may benefit from ANT-DBS the most. Further investigations are required to determine optimal parameters of stimulation.
\end{abstract}

RÉSUMÉ : Stimulation du noyau antérieur du thalamus dans les cas d'épilepsie : résultats d'une expérience réalisée au Canada. Objectif : L'étude visait à décrire l'expérience de la stimulation cérébrale profonde du noyau antérieur du thalamus (SCP-NAT) dans le traitement de l'épilepsie, réalisée dans un centre spécialisé au Canada. Méthode : Ont été retenus dans l'étude tous les patients qui avaient subi une intervention de pose d'électrodes en vue de la SCP-NAT, entre 2013 (première expérience au centre des auteurs) et 2020. Ces patients souffraient d'épilepsie réfractaire au traitement et n'étaient pas candidats à la résection de la zone épileptogène, sans compter que la stimulation du nerf vague (SNV) n'avait pas donné les résultats escomptés. La fréquence mensuelle des crises d'épilepsie au départ a été calculée au cours des 3 mois précédant la SNV, puis à différents moments dans le temps, soit 3 mois avant la SCP-NAT, et au bout de 3, 6, 12, 24, 36, 48, 60 et 72 mois après la pose du dispositif de SCP-NAT. Chaque fois, la fréquence des crises d'épilepsie était comparée à celle notée au départ. Résultats : Six patients ont été soumis au traitement par la SCP-NAT : 3 (50 \%) souffraient d'épilepsie multifocale; 1 (16,6\%), d'épilepsie focale; et 2 (33,4\%), d'épilepsie focale et généralisée. Une réduction de $>50 \%$ du nombre de crises d'épilepsie a été observée durant le suivi à long terme chez 2 patients atteints d'épilepsie multifocale; par contre, aucune n'a été observée chez 3 patients $(50 \%): 2$ atteints d'épilepsie focale et généralisée, et 1 autre, d'épilepsie focale. La chirurgie ou la pose du dispositif n'ont pas provoqué d'effets indésirables. Deux patients $(33,3 \%)$ ont cependant éprouvé des céphalées légères et passagères par suite des stimulations. Conclusion : La SCP-NAT s'est révélée un traitement sûr et efficace de l'épilepsie focale rebelle. L'expérience donne à penser que les patients souffrant d'épilepsie multifocale en raison d'une lésion régionale seraient les plus susceptibles de profiter du traitement par la SCP-NAT. Il faudrait toutefois pousser la recherche afin de déterminer les meilleurs paramètres possible de la stimulation.

Keywords: Epilepsy, Deep brain stimulation, Thalamus, Epilepsy surgery

doi:10.1017/cjn.2020.230

Can J Neurol Sci. 2021; 48: 469-478

Epilepsy is a common chronic neurologic disease, which has an estimated global prevalence of $1 \%$ and an incidence of 68 per 100,000 people per year. ${ }^{1,2}$ In 2010, The Global Burden of Disease Study found epilepsy to be the neurological disorder with the second most significant burden of disease, preceded only by headache and migraine. ${ }^{3}$ Approximately two-thirds of patients with epilepsy will have good seizure control with antiseizure drugs (ASDs), but the remaining third will have a therapyresistant condition. ${ }^{1,4}$

Therapy-resistant epilepsy (TRE) is defined by the International League Against Epilepsy (ILAE) as a failure to achieve a sustained seizure control after two appropriately chosen, scheduled, and

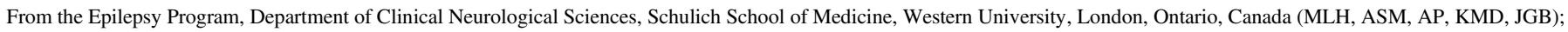
and Neuro-Epidemiology Unit, Schulich School of Medicine, Western University, London, Ontario, Canada (JGB)

Received May 29, 2020. Final Revisions Submitted September 22, 2020. Date of Acceptance October 10, 2020.

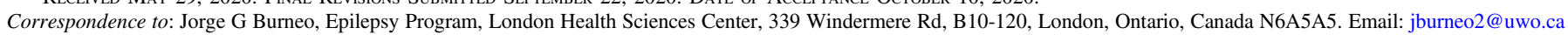




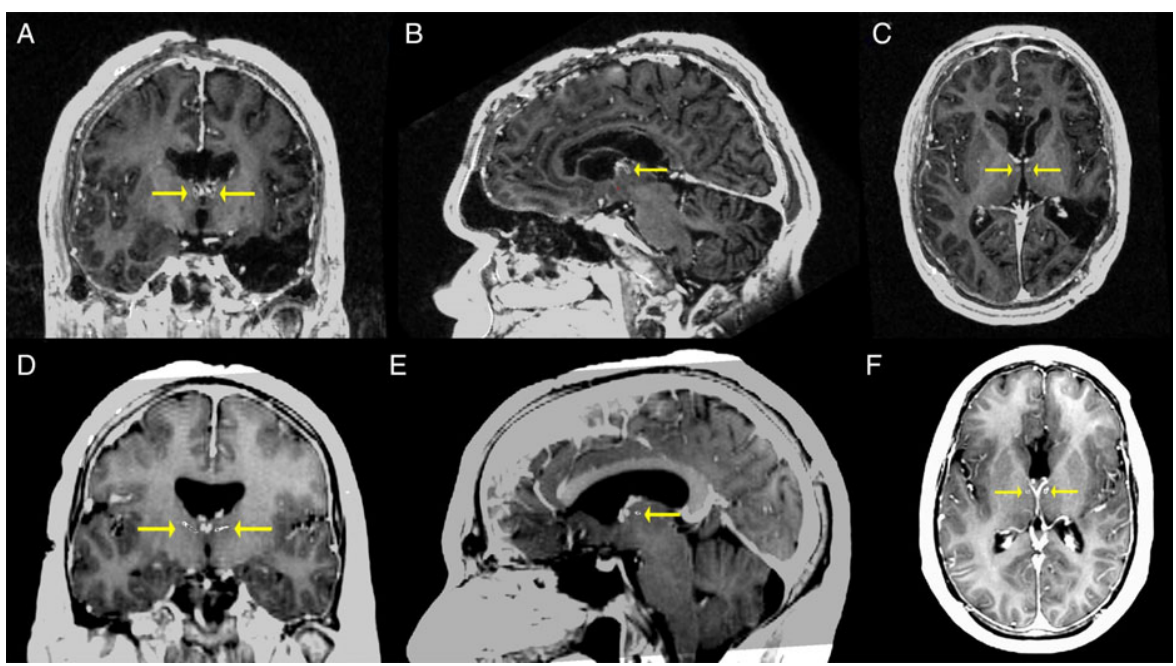

Figure 1: Contrast magnetic resonance imaging depicting patient \#6 $(A, B, C)$ and patient \#2 $(D, E, F)$.

tolerated ASDs, given as monotherapy or in combination. ${ }^{5}$ For patients with TRE, several studies including a meta-analysis described a significant benefit for surgical treatment, particularly for those affected by temporal lobe epilepsy. ${ }^{6}$ However, the effectiveness of surgery decreases for patients who have either extratemporal epileptogenic foci or no structural lesions. 6,7

When surgery has failed or is not indicated (either because of multiple epileptogenic foci, a widespread epileptogenic zone, a focus in an eloquent region or ongoing medical contraindications for surgery), neurostimulation becomes an important treatment alternative. ${ }^{6,8}$

Vagus nerve stimulation (VNS), deep brain stimulation (DBS), and responsive neurostimulation (RNS) have emerged in the last two decades as important treatment alternatives for TRE. DBS involves the delivery of continuous electrical stimulation (open loop) through implanted electrodes connected to a source (pulse generator), ${ }^{9}$ targeting different areas within the central nervous system. These areas have included the anterior nucleus of the thalamus (ANT), hippocampi (HC), centromedian nuclei of the thalamus, subthalamic nuclei, cerebellum, and posterior hypothalamus. The accumulated evidence supports the effectiveness of stimulation of the ANT and $\mathrm{HC}$ in patients with TRE. But, open-loop HC-DBS has largely been supplanted by the use of the RNS, particularly in the USA. ${ }^{9}$

Our center started implanting ANT-DBS in 2013 (approved in Canada in 2012). The purpose of this manuscript is to describe our experience on the use of ANT-DBS in patients with TRE.

\section{Methods}

We included all patients with epilepsy who underwent ANTDBS at our institution between 2013 and 2020.

In our center, candidates for ANT-DBS have been those with TRE (focal, multifocal, and/or generalized), have not been candidates for resective surgery - or it was unsuccessful, and have failed treatment with VNS.

Each patient underwent frame-based, stereotactic implantation of DBS leads (model 3389; Medtronic Inc., Minneapolis,
Minnesota, USA). Since the thalamic target for epilepsy is a much smaller target than the thalamic target usually used for tremor, there would be no advantage in choosing an electrode with a 10.5 -mm span (3387) when the nucleus only has a height of 3 to $4 \mathrm{~mm}$. The 3389 electrode measures $7.5 \mathrm{~mm}$ from end to end and will cover most of the nucleus, all 4 contacts are potentially available for stimulation. ${ }^{10}$ The implantable pulse generator (model 37601 or 37602; Medtronic Inc., Minneapolis, Minnesota, USA) was implanted during the same procedure. Target planning was based on anatomical identification of the ANT and the relevant adjacent white matter structures (mammillothalamic tract, external medullary lamina) on a preoperative contrast-enhanced volumetric T1 image and high-resolution T2-weighted axial and coronal $1.5 \mathrm{~T}$ magnetic resonance imaging (MRI) slices. After application of the stereotactic frame (Leksell Frame - Elekta, Stockholm, Sweden) on the morning of surgery, a contrast-enhanced volumetric computerized tomography (CT) scan was obtained. The CT and MRI images were fused to determine the stereotactic coordinates. For research purposes, electroencephalography (EEG) monitoring with simultaneous stimulation through DBS contacts was carried out in three patients, and microelectrode recording was carried out in three patients. These data were not used to determine the site of electrode implantation.

Postoperative head MRI ( 24 or 48 hours after implantation) was carried out in all patients to confirm electrode positioning. The contacts used for the stimulation were selected according to images (contacts placed closed to the center of ANT) and the impedance sensed on follow-up (Figure 1).

The VNS device was removed in patients \#1 and \#6 and was turned OFF in the remaining four patients.

In addition to the demographic and clinical variables, we assessed seizure outcome at 3, 6, 12, 24, 36, 48, 60, and 72 months post-implantation regarding a baseline frequency which was defined as the seizure frequency per month over a 3-month period before VNS implantation.

Nonparametric variables were reported as median and interquartile range (IQR) for continuous variables, and number value and percentage for categorical variables. 
Absolute values for monthly seizure frequency, detailed for every type of seizure per patient at each point in time, was carried out.

The variation in seizure frequency was obtained comparing the difference between seizure frequency at specific prior stablished point in time and the baseline seizure frequency, regarding this last one with subsequent percentual expression.

The epilepsy types were grouped according to the last ILAE classification of epilepsy of $2017^{11}$ : focal epilepsy, generalized epilepsy, combined generalized and focal (understood as independent seizure onset: focal or generalized, this is not referred to seizures with focal onset and subsequent progression to bilateral tonic-clonic (BTC) seizures), and unknown epilepsy (not used in our series). A special consideration and independent classification were done for multifocal epilepsies (formerly included in the focal epilepsy's group according to ILAE). This epilepsy was operationally defined as that with equal or more than three epileptic foci in different lobes.

This study was approved by the Western University Health Sciences Research Ethics Board (HSREB).

\section{Results}

\section{Demographic, Clinical, and Neurostimulation Findings}

Six patients underwent ANT-DBS implantation since 2013. The median age was 34 years (IQR 29-39), five patients were male $(83 \%)$, and the median age at epilepsy onset was 14 years (IQR 5.5-15.5). The median duration of epilepsy was 25 years (IQR 21-27). The median age at DBS implantation was 30.5 years (IQR 24-33) (Table 1).

Three $(50 \%)$ patients had multifocal epilepsy, one (16.6\%) had focal epilepsy due to extensive polymicrogyria over the perisylvian region (failed previous parietal corticectomy and persistent epileptogenic focus overlapped with primary motor cortex), and two (33.4\%) had combined generalized and focal epilepsy. Five patients had MRI lesions as shown in Table 1. All patients tried numerous ASDs, with a mean of 6.5 (IQR 5-9).

All the patients were admitted to the epilepsy monitoring unit (EMU) and underwent scalp EEG recordings. Five patients (83\%) underwent intracranial electrodes; the patient \#3 underwent stereoelectroencephalography (SEEG) 10 years after subdural recording. Patient \#4 did not have intracranial studies due to the multifocal nature of his/her non-lesional epilepsy since the beginning. All the patients had at least one extratemporal epileptic focus (Table 1).

The DBS parameters are detailed in Table 2 as well as changes made to the ASD regimens. The time for turning ON the device was quite variable. For patient \#1, the DBS was turned ON within 72 hours. For patient \#6, the DBS was turned ON 1 week after implantation. For patients \#2 and \#3, the DBS was turned ON 1 month after implantation. The patients \#4 and \#5 had a DBS stimulation onset 2 months after implantation.

Patient \#2 had an initial voltage of $0.5 \mathrm{~V}$ because he developed some mild and temporary device-related side effects. Patient \#4 had an initial voltage of $0.5 \mathrm{~V}$ when the device was turned $\mathrm{ON}$ as a precaution due to his/her known tendency to develop status epilepticus and prolonged BTC with severe injuries associated.

\section{Seizure Outcomes and Long-Term Follow-Up}

The mean follow-up time after DBS implantation was 4.9 years (IQR 3-6) (Table 2). Two patients (\#1 and \#2) showed a seizure reduction $>50 \%$. These two patients had a sustained progressive improvement (Table 3 and 4). Patient \#1 reached 5 months seizure freedom around the 60 months' follow-up. Patient \#2 had a decrease in more than $50 \%$ of seizure frequency after the 24 months' follow-up. These two patients have multifocal epilepsy with lesional MRI.

The follow-up was based on seizure frequency (no interictal activity). Routine EEG recordings were not performed after surgery except for those patients who had persistent seizures or clinical events.

In terms of interictal activity, patient \#1 showed a clear reduction of bifrontal spikes but persistent right temporal and left posterior temporal spikes. Interictal activity prior implantation showed multiple independent spike foci, prominent over the left hemisphere and right frontal-temporal region.

Patient \#3 was admitted to our EMU 2-3 years after DBS implantation. Small amount of left central parietal spikes was reported, mainly during sleep. Focal slowing over left central parietal region without spikes was reported the most of the time. Recordings of EMU admissions prior to DBS implantation showed daily large amount (during wakefulness and sleep) of central sagittal spikes.

Patient \#5 had an admission a year and a half after DBS insertion (battery was found depleted by that time). There were not changes in interictal activity regarding recordings prior to DBS.

Patient \#6 had several admissions after DBS implantation due to persistent seizures in a context of Lennox-Gastaut syndrome. Similar interictal activity was seen in recordings prior and after DBS implantation.

One patient (\#4) died 18 months after implantation due to sudden unexpected death in epilepsy (SUDEP). Assessment at 3 and 12 months after DBS implantation showed seizure reduction of $25 \%$ and $13 \%$, respectively (Table 3 and 4). This patient had combined generalized epilepsy with normal MRI.

Two patients (\#3 and \#6) have not shown seizure reduction after implantation, rather, they showed an increase in seizure frequency at 48 months (295\%) and at 60 months (275\%), respectively (Table 3 and 4). Patient \#3 had focal epilepsy and patient \#6 had combined focal and generalized epilepsy.

The patient \#5 showed an initial tendency to improve his/her seizure frequency, and the highest reduction was reached at 24 months. Between 24 and 36 months, this patient had an increment of focal impaired-awareness seizures and resumption of tonic seizures at least one per month (Table 3 and 4). There were no side effects documented. Patient's father requested turning OFF the device which was done at 38 months of follow-up. This patient has multifocal epilepsy.

Figure 2 represented the evolution of seizure frequency for patients with multifocal epilepsy until 36 months of follow-up.

\section{Side Effects}

Patients did not have surgical-related (hemorrhage and surgical wound infection) or device-related (malfunctioning, electrodes fracture, and misplacement leads) side effects.

Patient \#2 developed a throbbing headache, neck pain, and lightheadedness episodes with position changes since the surgery. Those effects disappeared gradually over the next 2 months and were considered device-related side effects. According to the 
Table 1: Demographic and clinical characteristics of patients prior to ATN-DBS implantation

\begin{tabular}{|c|c|c|c|c|c|c|c|c|c|c|}
\hline Patient & Age (years) & Sex & Handedness & $\begin{array}{c}\text { Age of epilepsy onset } \\
\text { (years) }\end{array}$ & Comorbidities & Epilepsy types & $\begin{array}{c}\text { Intracranial } \\
\text { electrodes }\end{array}$ & Ictal (seizures onset) & Interictal & MRI \\
\hline$\# 1$ & 38 & $\mathrm{~F}$ & $\mathrm{R}$ & 14 & $\begin{array}{l}\text { Papillary site cancer - } \\
\text { thyroidectomy }\end{array}$ & Multifocal & Subdural & $\begin{array}{l}\text { L mesial temporal, L } \\
\text { neocortical and posterior } \\
\text { temporal, Loccipital mesia }\end{array}$ & $\begin{array}{l}\text { MISF: Left hemisphere and right } \\
\text { temporal-frontal }\end{array}$ & Mild left hemisphere atrophy \\
\hline$\# 2$ & 38 & м & $\mathrm{R}$ & 16 & & Multifocal & \begin{tabular}{|l|} 
Simultaneous subdural and \\
depth
\end{tabular} & $\begin{array}{l}\text { R hemisphere, } \mathrm{L} \text { posterior } \\
\text { mesial temporal }\end{array}$ & $\begin{array}{l}R \text { frontal and central. Independent } \\
\text { bilateral temporal }\end{array}$ & Bilateral perysilvial polymicrogyria \\
\hline$\# 3$ & 35 & M & $\mathrm{R}$ & 9 & Remote viral encephalitis & Focal epilepsy & \begin{tabular}{|l} 
Subdural and depth \\
electrodes (SEEG)
\end{tabular} & \begin{tabular}{|l} 
L frontal and parietal \\
(primary motor and \\
sensory; SSMA too)
\end{tabular} & L central-parietal sagital, $\mathrm{L}$ temporal & Left frontoparietal cortical dysplasia \\
\hline$\# 4$ & 33 & м & $\mathrm{R}$ & 2 & $\begin{array}{l}\text { Remote traumatic epidural } \\
\text { hematoma }\end{array}$ & $\begin{array}{l}\text { Combined generalized and } \\
\text { focal }\end{array}$ & No & R frontal and generalized & MISF and generalized polyspikes & \\
\hline \#5 & 30 & м & $\mathrm{R}$ & 15 & \begin{tabular}{|l} 
Acute lymphoblastic \\
leukemia - radiotherapy
\end{tabular} & Multifocal & Subdural & $\begin{array}{l}\text { L parietal, L lateral } \\
\text { occipital, L temporal } \\
\text { convexity, L mesial } \\
\text { frontal, query generalized. }\end{array}$ & $\begin{array}{l}\text { MISF, maximum independent bilateral } \\
\text { posterior head }\end{array}$ & $\begin{array}{l}\text { Focal encephalomalacia }(\mathrm{R} \text { posterio } \\
\text { parietal). Corpus callosotomy }\end{array}$ \\
\hline$\# 6$ & 25 & м & $\mathrm{R}$ & 0.56 & Lennox-Gastaut syndrome & $\begin{array}{l}\text { Combined generalized and } \\
\text { focal }\end{array}$ & Subdural & $\begin{array}{l}\mathrm{R} \text { hemisphere, } \mathrm{L} \text { temporal } \\
\text { occipital, generalized (low } \\
\text { voltage, fast activity). }\end{array}$ & MISF, maximum right posterior head. & $\begin{array}{l}\text { R extensive frontal-temporal-parietal } \\
\text { encephalomalacia }\end{array}$ \\
\hline \multirow[t]{2}{*}{ Patient } & \multirow{2}{*}{$\begin{array}{c}\text { Otder imaging } \\
\text { study }\end{array}$} & \multirow{2}{*}{$\begin{array}{c}\text { Number of ASD } \\
\text { failed }\end{array}$} & \multirow{2}{*}{$\begin{array}{c}\text { Number of ASD } \\
\text { (last schedule) }\end{array}$} & \multirow[b]{2}{*}{$\begin{array}{l}\text { Previous palliative } \\
\text { surgery }\end{array}$} & \multirow{2}{*}{$\begin{array}{l}\text { Age (years) of VNS } \\
\text { implant. }\end{array}$} & \multicolumn{5}{|c|}{ VNS Settings } \\
\hline & & & & & & \begin{tabular}{|l}
$\begin{array}{l}\text { Output current mA } \\
\text { (min-max) }\end{array}$ \\
\end{tabular} & Frequency $\mathbf{H z}$ & Pulse $(\mu s)$ & Signal ON (seconds) & Signal OFF (minutes) \\
\hline$\# 1$ & & 8 & 3 & & 28 & $0,25-1,75$ & 30 & 250 & 30 & 3 \\
\hline$\# 2$ & & 5 & 4 & & 30 & $0,25-3$ & 30 & 500 & 30 & 3 \\
\hline \#3 & \begin{tabular}{|c|} 
SPECT: left post- \\
temporal lobe \\
(inferior to \\
corticectomy)
\end{tabular} & 5 & 3 & \begin{tabular}{|l|} 
Corticectomy: left parietal \\
\end{tabular} & 27 & $0,25-2,75$ & 30 & 500 & 30 & 3 \\
\hline$\# 4$ & & 5 & 3 & & 25 & $0,25-3,5$ & 40 & 250 & 60 & 3 \\
\hline \#5 & & 8 & 4 & $\begin{array}{c}\text { Partial and posterior complete } \\
\text { callosostomy }\end{array}$ & 21 & $0,25-3$ & 30 & 500 & 30 & 3 \\
\hline \#6 & & 10 & 3 & $\begin{array}{l}\text { R. temporal and parietal } \\
\text { resection. R frontal } \\
\text { corticectomy. Complete } \\
\text { callosotomy. }\end{array}$ & 8 & $0,25-2$ & 30 & 250 & 30 & 3 \\
\hline
\end{tabular}

R: right; L: left; MISF: Multiple independent spike foci; SSMA: Supplementary sensory-motor area; SEEG: Stereoelectroencephalography; MRI: Magnetic Resonance Imaging; SPECT: SinglePhoton Emission Computerized Tomography; VNS: Vagus Nerve Stimulator. 


\section{Table 2: Description of anti-seizure treatment received and ATN-DBS parameters}

\begin{tabular}{|c|c|c|c|c|c|c|c|c|c|c|c|}
\hline \multirow{2}{*}{ Patient } & \multirow{2}{*}{$\begin{array}{c}\text { Age DBS } \\
\text { implant. } \\
\text { (years) } \\
\end{array}$} & \multicolumn{9}{|c|}{ Antiseizure drugs (mg) and DBS parameters $(*)$} & \multirow{2}{*}{ Follow-up $(\mathbf{Y})$} \\
\hline & & At implantation & 3 months & 6 months & 12 months & 24 months & 36 months & 48 months & 60 months & 72 months & \\
\hline \multirow[t]{2}{*}{$\# 1$} & 32 & $\begin{array}{l}\text { TOP } 100 \text { BID } \\
\text { OXC } 300 \text { BID } \\
\text { LCS } 250-200\end{array}$ & $\begin{array}{l}\text { TOP } 100 \text { BID } \\
\text { OXC } 450 \text { BID } \\
\text { LCS } 200 \text { BID }\end{array}$ & $\begin{array}{l}\text { TOP } 100 \text { BID } \\
\text { OXC } 450 \text { BID } \\
\text { LCS } 200 \text { BID }\end{array}$ & $\begin{array}{l}\text { TOP } 100 \text { BID } \\
\text { OXC } 450 \text { BID } \\
\text { LCS } 200 \text { BID } \\
\text { PeRM } 8 \text { HS } \\
\end{array}$ & $\begin{array}{l}\text { TOP } 100 \text { BID } \\
\text { OXC } 450 \text { BID } \\
\text { LCS } 200 \text { BID } \\
\text { PeRM } 10 \text { HS } \\
\end{array}$ & $\begin{array}{l}\text { TOP } 100 \text { BID } \\
\text { OXC } 450 \text { BID } \\
\text { LCS } 200 \text { BID } \\
\text { PeRM } 10 \text { HS } \\
\end{array}$ & $\begin{array}{l}\text { TOP } 100 \text { BID } \\
\text { OXC } 450 \text { BID } \\
\text { LCS } 200 \text { BID } \\
\text { PeRM } 10 \text { HS } \\
\end{array}$ & $\begin{array}{l}\text { TOP } 100 \text { BID } \\
\text { OXC } 450 \text { BID } \\
\text { LCS } 200 \text { BID } \\
\text { PeRM } 10 \text { HS } \\
\end{array}$ & \begin{tabular}{|l|} 
TOP 100 BID \\
OXC 450 BID \\
LCS 200 BID \\
PeRM 10 HS \\
\end{tabular} & 6 \\
\hline & & & $4.3 \mathrm{~V}$ & $4.5 \mathrm{~V}$ & $4.8 \mathrm{~V}$ & $5 \mathrm{~V}$ & $5 \mathrm{~V}$ & $5 \mathrm{~V}(\mathrm{~L})-5.5 \mathrm{~V}(\mathrm{R})$ & $5.5 \mathrm{~V}$ & $5.6 \mathrm{~V}(\mathrm{R})-7 \mathrm{~V}(\mathrm{~L})$ & \\
\hline \multirow[t]{2}{*}{ \#2 } & 35 & $\begin{array}{l}\text { CBZ 200 BID TOP } \\
125 \text { BID LEV } 500 \\
\text { BID }\end{array}$ & $\begin{array}{l}\text { CBZ 200 BID TOP } \\
125 \text { BID LEV } 500 \\
\text { BID }\end{array}$ & $\begin{array}{l}\text { CBZ 200 BID TOP } \\
125 \text { BID LEV } 500 \\
\text { BID }\end{array}$ & $\begin{array}{l}\text { CBZ 200 BID TOP } \\
125 \text { BID BVL } 100 \\
\text { BID CLZ } 10 \text { HS }\end{array}$ & $\begin{array}{l}\text { CBZ } 100 \text { BID TOP } \\
125 \text { BID BVL } 100 \\
\text { BID }\end{array}$ & $\begin{array}{l}\text { TOP } 150 \text { BID } \\
\text { BVL } 100 \text { BID }\end{array}$ & & & & 3 \\
\hline & & & $1 \mathrm{~V}(\mathrm{R})-1.2(\mathrm{~L})$ & $3 \mathrm{~V}$ & $4 \mathrm{~V}$ & $5.4(\mathrm{R})-5 \mathrm{~V}(\mathrm{~L})$ & $5.6 \mathrm{~V}(\mathrm{R})-5.2 \mathrm{~V}(\mathrm{~L})$ & & & & \\
\hline \multirow[t]{2}{*}{$\# 3$} & 30 & $\begin{array}{l}\text { LEV } 1500 \text { BID } \\
\text { OXC } 1200 \\
\text { BID LCS } 200-150\end{array}$ & $\begin{array}{l}\text { LEV } 1500 \text { BID } \\
\text { OXC } 1200 \text { BID } \\
\text { LCS } 200-150\end{array}$ & $\begin{array}{l}\text { LEV } 1500 \text { BID } \\
\text { OXC } 1200 \text { BID } \\
\text { LCS } 200-150\end{array}$ & $\begin{array}{l}\text { LEV } 1500 \text { BID } \\
\text { OXC } 1200 \text { BID } \\
\text { LCS } 200-150\end{array}$ & $\begin{array}{l}\text { OLEV } 1500 \text { BID } \\
\text { LCS 200-150 } \\
\text { PeRM 8mg HS }\end{array}$ & $\begin{array}{l}\text { LEV } 1500 \text { BID } \\
\text { LCS 200-150; } \\
\text { PRM 500 BID }\end{array}$ & $\begin{array}{l}\text { LEV 2000-1250 } \\
\text { LCS 200-150 } \\
\text { PRM } 250 \text { HS CLB } \\
10 \mathrm{mg}\end{array}$ & & & 4 \\
\hline & & & $5.5 \mathrm{~V}$ & $6 \mathrm{~V}$ & $7 \mathrm{~V}$ & $8 \mathrm{~V}$ & $8.5 \mathrm{~V}$ & $8.5 \mathrm{~V}$ & & & \\
\hline \multirow[t]{2}{*}{ \#4 } & 31 & $\begin{array}{l}\text { LMT } 200 \text { BID } \\
\text { LCS } 200 \text { BID ESL } \\
800 \text { HS Cannabis } \\
3 \mathrm{~g}\end{array}$ & \begin{tabular}{|l|} 
LMT 200 BID \\
LCS 200 BID ESL \\
800 HS Cannabis \\
$3 \mathrm{~g}$ \\
\end{tabular} & $\begin{array}{l}\text { LMT } 200 \text { BID } \\
\text { LCS } 200 \text { BID ESL } \\
800 \mathrm{mg} \text { HS }\end{array}$ & $\begin{array}{l}\text { LMT } 200 \text { BID } \\
\text { LCS } 200 \text { BID ESL } \\
800 \text { HS }\end{array}$ & $\begin{array}{l}\text { SUDEP } \\
\text { (18 months after } \\
\text { implantation) }\end{array}$ & SUDEP & & & & 1 \\
\hline & & & $0.5 \mathrm{~V}$ & $2 \mathrm{~V}$ & $3.5 \mathrm{~V}(\mathrm{R})-3.7(\mathrm{~L})$ & & & & & & \\
\hline \multirow[t]{2}{*}{$\# 5$} & 25 & $\begin{array}{l}\text { LEV } 500 \text { BID PHT } \\
\text { 200-250 CBZ } 500 \\
\text { BID }\end{array}$ & $\begin{array}{l}\text { LEV } 500 \text { BID PHT } \\
\text { 200-250 CBZ } 500 \\
\text { BID }\end{array}$ & $\begin{array}{l}\text { LEV } 500 \text { BID PHT } \\
\text { 200-250 CBZ } 500 \\
\text { BID }\end{array}$ & $\begin{array}{l}\text { LEV } 500 \text { BID PHT } \\
\text { 200-250 ESL } 1200 \\
\text { HS }\end{array}$ & $\begin{array}{l}* * \text { LEV } 500 \text { BID } \\
\text { PHT } 200-150 \\
\text { OXC } 450 \text { BID } \\
\text { ESL } 1000 \mathrm{HS}\end{array}$ & $\begin{array}{l}\text { LEV } 500 \text { BID PHT } \\
200-150 \text { OXC } 450 \\
\text { BID ESL } 1000 \text { HS }\end{array}$ & $\begin{array}{l}\text { LEV } 750 \text { BID PHT } \\
200-150 \text { OXC } 450 \\
\text { BID RUF } 1600 \\
\text { BID FLB } 600 \text { BID }\end{array}$ & & & 4 \\
\hline & & & $5.5 \mathrm{~V}$ & $6.5 \mathrm{~V}$ & $8 \mathrm{~V}$ & $5 \mathrm{~V}(\mathrm{R})-3.5 \mathrm{~V}(\mathrm{~L})$ & $6 \mathrm{~V}(\mathrm{R})-5 \mathrm{~V}(\mathrm{~L})$ & & & & \\
\hline \multirow[t]{2}{*}{$\# 6$} & 21 & $\begin{array}{l}\text { PHT } 180 \text { BID } \\
\text { VAC 500-125-500 } \\
\text { CLB } 5 \text { HS }\end{array}$ & \begin{tabular}{|l} 
PHT 200-180 \\
VAC 500-125-500 \\
CLB 7.5 HS
\end{tabular} & $\begin{array}{l}\text { PHT } 180 \text { BID } \\
\text { VAC } 500-125-500 \\
\text { CLB } 7.5 \text { HS CBD } \\
14 \text { g + K.D. }\end{array}$ & $\begin{array}{l}\text { PHT } 180 \text { BID } \\
\text { VAC } 500-125-500 \\
\text { CLB } 7.5 \text { HS CBD } \\
14 \mathrm{~g}+\text { K.D. }\end{array}$ & $\begin{array}{l}\text { PHT } 180 \text { BID } \\
\text { VAC } 500-375-500 \\
\text { CLB } 10 \mathrm{HS}\end{array}$ & $\begin{array}{l}\text { PHT } 180 \text { BID } \\
\text { PeRM } 8 \text { HS CLB } \\
20 \text { HS }\end{array}$ & $\begin{array}{l}\text { PHT 180-155 CLB } \\
5-20\end{array}$ & $\begin{array}{l}\text { PHT 180-175 } \\
\text { CLB 20 HS } \\
\text { Battery OFF }\end{array}$ & & 5 \\
\hline & & $5 \mathrm{~V}$ & $5.8 \mathrm{~V}$ & $6.2 \mathrm{~V}$ & $7 \mathrm{~V}$ & $8 \mathrm{~V}$ & $8 \mathrm{~V}$ & $8 \mathrm{~V}$ & & & \\
\hline
\end{tabular}

Y: Years; DBS: deep brain stimulation.

*Deep Brain Stimulation Parameters: $145 \mathrm{~Hz}$; pulse width of $90 \mu$ seconds; cycle 1 min ON and 5 min OFF.

**Switching from ESL to OXC was tried, but once ESL was weaned off, seizures increased and ESL was resumed.

CDBS Cycle mode was switched to continuous (at 18 months).

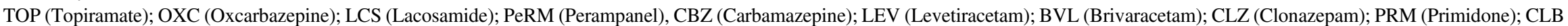

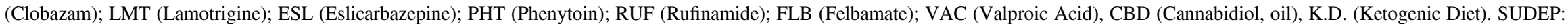

Sudden Unexpected Death in Epilepsy. 
Table 3: Clinical outcome following ATN-DBS implantation

\begin{tabular}{|c|c|c|c|c|c|c|c|c|c|c|c|}
\hline \multirow[b]{2}{*}{ Patient } & \multirow[b]{2}{*}{ Seizure type } & \multicolumn{10}{|c|}{ Seizure frequency (per month) } \\
\hline & & Baseline & Af. VNS & 3m Af. DBS & 6m Af. DBS & $\begin{array}{c}\text { 12m Af. } \\
\text { DBS }\end{array}$ & $\begin{array}{c}24 m \text { Af. } \\
\text { DBS }\end{array}$ & $\begin{array}{c}\text { 36m Af. } \\
\text { DBS }\end{array}$ & $\begin{array}{l}\text { 48m Af. } \\
\text { DBS }\end{array}$ & $\begin{array}{c}\text { 60m Af. } \\
\text { DBS }\end{array}$ & $\begin{array}{c}\text { 72m Af. } \\
\text { DBS }\end{array}$ \\
\hline \multirow[t]{6}{*}{$\# 1$} & $\begin{array}{l}\text { Focal aware } \\
\text { nonmotor }\end{array}$ & 10 & 10 & 3 & 4 & 3 & 1 & 1 & 2 & 0 & 0 \\
\hline & Focal aware motor & & & & & & & & & & \\
\hline & Focal with LOA & 3 & & 3 & & & 1 & & 0 & 0 & 2 \\
\hline & BTC & 1 & 6 & & 4 & 2 & & 1 & & & \\
\hline & Generalized & & & & & & & & & & \\
\hline & Total & 14 & 16 & 6 & 8 & 5 & 2 & 2 & 2 & 0 & 2 \\
\hline \multirow[t]{6}{*}{$\# 2$} & $\begin{array}{l}\text { Focal aware } \\
\text { nonmotor }\end{array}$ & 19 & 28 & 18 & 21 & 19 & 8 & 6 & & & \\
\hline & Focal aware motor & & & & & & & & & & \\
\hline & Focal with LOA & 5 & 5 & 5 & 2 & 6 & 4 & 2 & & & \\
\hline & BTC & 1 & & & & & & & & & \\
\hline & Generalized & & & & & & & & & & \\
\hline & Total & 25 & 33 & 23 & 23 & 25 & 12 & 8 & & & \\
\hline \multirow[t]{6}{*}{$\# 3$} & $\begin{array}{l}\text { Focal aware } \\
\text { nonmotor }\end{array}$ & 40 & 30 & 39 & 40 & 118 & 559 & 168 & 161 & & \\
\hline & Focal aware motor & & & & & & & & & & \\
\hline & Focal with LOA & & 1 & & & 4 & & & & & \\
\hline & BTC & 1 & 1 & 1 & 2 & 1 & 1 & 0 & 1 & & \\
\hline & Generalized & & & & & & & & & & \\
\hline & Total & 41 & 32 & 40 & 42 & 123 & 560 & 168 & 162 & & \\
\hline \multirow[t]{6}{*}{$\# 4$} & $\begin{array}{l}\text { Focal aware } \\
\text { nonmotor }\end{array}$ & & & & & & \multirow[t]{6}{*}{$\begin{array}{l}\text { SUDEP at } \\
18 \text { months }\end{array}$} & & & & \\
\hline & Focal aware motor & & & & & & & & & & \\
\hline & Focal with LOA & & & & & & & & & & \\
\hline & BTC & 8 & 8 & 6 & 13 & 7 & & & & & \\
\hline & Generalized & & & & & & & & & & \\
\hline & Total & 8 & 8 & 6 & 13 & 7 & & & & & \\
\hline \multirow[t]{6}{*}{$\# 5$} & \begin{tabular}{|l} 
Focal aware \\
nonmotor
\end{tabular} & & & & & & & & & & \\
\hline & Focal aware motor & & & & & & & & & & \\
\hline & Focal with LOA & 105 & 110 & 114 & 155 & 126 & 63 & 121 & 78 & & \\
\hline & BTC & 1 & & & & & & & & & \\
\hline & Generalized (tonic) & & & & 2 & 2 & & 1 & 1 & & \\
\hline & Total & 106 & 110 & 114 & 157 & 128 & 63 & $113 \dagger$ & 85.3 & & \\
\hline \multirow[t]{6}{*}{$\# 6$} & $\begin{array}{l}\text { Focal aware } \\
\text { motor } * * *\end{array}$ & & & & & & & & & & \\
\hline & \begin{tabular}{|l} 
Focal aware \\
nonmotor
\end{tabular} & & & & & & & & & & \\
\hline & Focal with LOA & 30 & 20 & 30 & 45 & 16 & 45 & 45 & 30 & 90 & \\
\hline & BTC & 1 & & & & & & & & & \\
\hline & $\begin{array}{l}\text { Generalized (tonic } \\
+ \text { tonic-clonic) }\end{array}$ & 1 & 30 & 30 & 31 & 9 & 8 & 5 & 24 & 30 & \\
\hline & Total & 32 & 50 & 60 & 76 & 25 & 53 & 50 & 54 & 120 & \\
\hline
\end{tabular}

$\dagger$ DBS turned off right after 38 months' follow-up.

m: months; LOA: Loss of awareness; BTC: Bilateral tonic-clonic. VNS: Vagus Nerve Stimulator; DBS: Deep Brain Stimulator; SUDEP: Sudden Unexpected Death in Epilepsy. 
Table 4: Seizure frequency following ATN-DBS implantation

\begin{tabular}{l|c|c|c|c|c|c|c|c|c}
\hline \multirow{2}{*}{ Patient } & \multirow{2}{*}{ After VNS } & \multicolumn{7}{|c}{ Seizure frequency after DBS placement } \\
\cline { 3 - 10 } & & $\mathbf{3}$ months & $\mathbf{6}$ months & $\mathbf{1 2}$ months & $\mathbf{2 4}$ months & $\mathbf{3 6}$ months & $\mathbf{4 8}$ months & $\mathbf{6 0}$ months & $\mathbf{7 2}$ months \\
\hline$\# 1$ & $14 \%$ & $-57 \%$ & $-43 \%$ & $-64 \%$ & $-86 \%$ & $-86 \%$ & $-86 \%$ & $-100 \%$ & $-86 \%$ \\
\hline$\# 2$ & $32 \%$ & $-8 \%$ & $-8 \%$ & $0 \%$ & $-52 \%$ & $-68 \%$ & & & \\
\hline$\# 3$ & $-22 \%$ & $-2 \%$ & $2 \%$ & $200 \%$ & $1266 \%$ & $310 \%$ & $295 \%$ & & \\
\hline$\# 4$ & $0 \%$ & $-25 \%$ & $63 \%$ & $-13 \%$ & & & & & \\
\hline$\# 5$ & $4 \%$ & $8 \%$ & $48 \%$ & $21 \%$ & $-41 \%$ & $7 \%$ & $*-20 \%$ & & \\
\hline$\# 6$ & $56 \%$ & $88 \%$ & $138 \%$ & $-22 \%$ & $66 \%$ & $56 \%$ & $69 \%$ & $275 \%$ & \\
\hline
\end{tabular}

VNS: Vagus Nerve Stimulator; DBS: Deep Brain Stimulator; SUDEP: Sudden Unexpected Death in Epilepsy.

Percentage of seizure variation: ((Fcm Tx $-\mathrm{Fcm} \mathrm{Tb}) / \mathrm{Fcm} \mathrm{Tb}) * 100$

Fcm: Frequency of seizures per month.

Tx: Cohort of time assessed (after VNS, 3 months after DBS, 6 months after DBS, 12 months after DBS, 24 months after DBS, 36 months after DBS, 48 months after DBS, 60 months after DBS, 72 months after DBS).

Tb: Baseline

*DBS turned off at 38 months' follow-up.

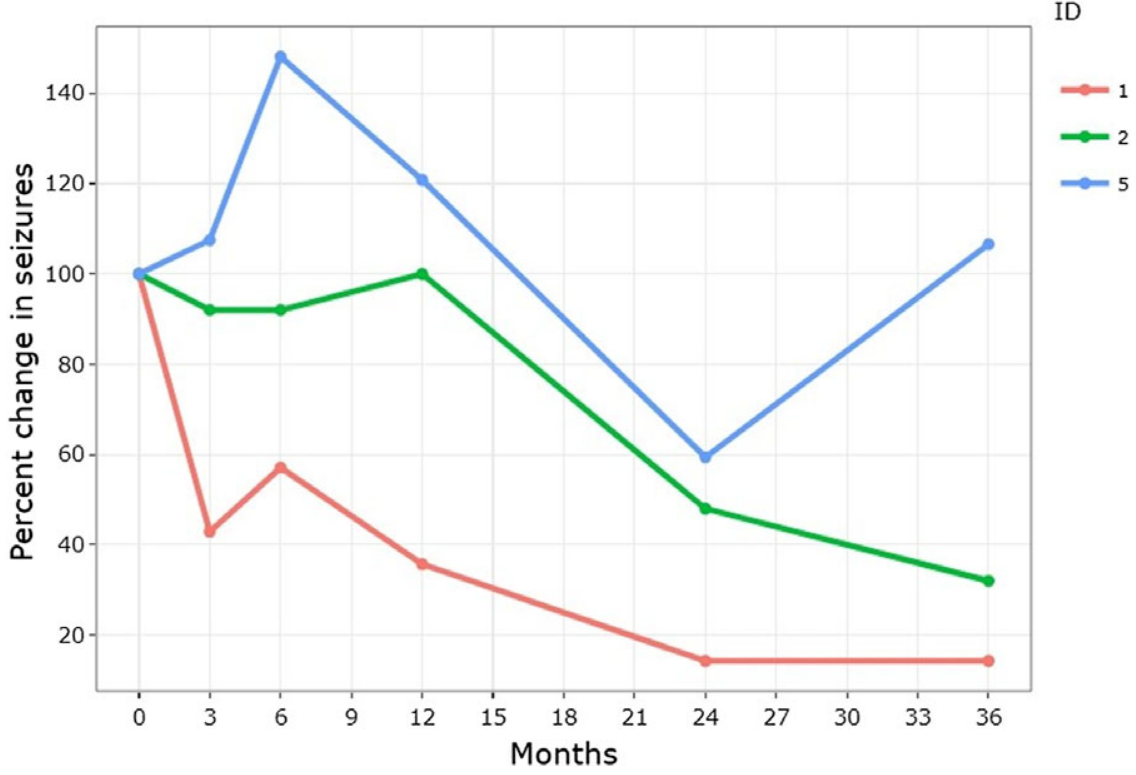

Figure 2: Multifocal epilepsy patients (\#1, \#2, and \#5): Percentage of seizure frequency changes within 36 months after DBS implantation.

Common Terminology Criteria for Adverse Events (CTCAE), this effect is considered in Nervous System Disorder (System Organ Class): headaches grade 2 adverse event.

Patient \#3 developed headaches right after turning ON the DBS. It was temporary and disappeared gradually within the next 4 weeks. It was considered functional-related side effects. According to the CTCAE, it is also considered in Nervous System Disorder (System Organ Class): headaches grade 2 adverse event.

Patient \#4 had a status epilepticus the fifth day after implantation. This was not considered a side effect due to his/her aggressive and poor controlled epilepsy (recurrent admissions due to status epilepticus) and a context of missing ASDs.
The patient \#3 had paroxysmal events of excessive nausea and vomiting right after battery replacement and the setting of cycling mode. These events were found to be seizures when the patient was admitted at the EMU. The switching from cycling mode to continuous mode stimulation resolved the problem.

One death occurred due to SUDEP, which was not considered to be device or stimulation-related.

\section{Discussion}

Despite the relatively small number of patients in this study, there was a clear reduction $(>50 \%)$ in seizure frequency in two patients with multifocal epilepsy and lesional MRI. 
The patient \#5 (included in the group of multifocal epilepsy) seemed to have an improvement at the end of the 24 months. However, along the first 12 months, the seizure frequency did not appear to get better. In this context, the battery was depleted and the decision was to turn OFF the DBS and see the evolution in the next 3 months. The patient had three BTC seizures (he/she used to have 1 or 2 per year) and similar amount of seizures with focal impaired awareness. Thus, it was decided to replace the battery and turn ON the DBS at month 18. This was followed by a decrease in the number of focal seizures but persistence of BTC. Antiseizure medication was modified during this second year (switching from eslicarbazepine to oxcarbazepine), but in this process, a better outcome was noticed when both drugs were used. In this setting, the assessment at 24 months showed an important improvement. It is not clear if this effect was due to the resumption of the DBS stimulation or due to ASD. The evolution during the next year was quite variable with improvements and impairments in terms of focal seizures but persistence of the BTC. Additional changes in medication were done (switching from levetiracetam to brivaracetam). At 36 months, the seizure frequency got worse and the device was turned OFF, as requested by patient's family, at 38 months' follow-up.

In the group of multifocal epilepsy, the main difference between patients \#1 and \#2 had an important seizure reduction $(>50 \%)$, and the patient \#5 was the etiology of the lesion. Patient \#1 (left hemisphere hypoplasia) and patient \#2 (bilateral perisylvian polymicrogyria) had more outlined regional lesions which might be related to malformations of cortical development. In contrast, patient \#5 had a multifocal epilepsy secondary to diffuse brain injury due to cranial radiotherapy (acute lymphoblastic leukemia in childhood) which on top of that showed an evolution in the clinical and electrographic features throughout the years.

Several open-label trials with small series of patients suggested the benefit of ANT-DBS for TRE. ${ }^{12-29}$ The Stimulation of the Anterior Nucleus of Thalamus for Epilepsy (SANTE) study was the first double-blind randomized trial which demonstrated significant efficacy and safety for the treatment of therapyresistant epilepsies, specifically focal temporal lobe epilepsies, and frontal lobe epilepsies to a lesser degree. ${ }^{30}$ However, the sample in the subgroup analysis for multifocal epilepsy was exceedingly small and nonconclusive. Otherwise, the long-term report of this study described a global reduction of seizure frequency around $69 \%$ after 5 years, as well as a $\geq 50 \%$ reduction in $68 \%$ of patients. $^{31}$

ANT-DBS has not been studied for multifocal epilepsy exclusively. Nevertheless, small series published in the last two decades included a few of these cases, with lesions on MRI in most of them. A tendency for good outcome $(>50 \%$ seizure reduction) was outlined. ${ }^{14-18}$

In our small series, the two patients with multifocal epilepsy who achieved a seizure reduction higher than $50 \%$ showed a sustained improvement over the years. Even though this could have been the effect of ASD changes or dose modifications (meds were stable in patient \#1 after the 12 months' follow-up; and drugs were slightly decreased in patient \#2), the effects of neurostimulation for epilepsy are cumulative and outcomes improve over time. ${ }^{30,31}$

Interestingly, the SANTE trial described at the end of 24 months of long-term follow-up, three patients had seizure frequency impairment due to increment of "simple partial seizures". ${ }^{30}$ In our series, the patient \#3 had a reduction in the number of BTC but was the exorbitant amount of focal-sensitive seizures that made this DBS therapy to be considered not successful and does not allow a statistical analysis.

Our small series found mild side effects device-related and functional-related but is known in the literature that paresthesias (18.2\%), implant side pain (10.9\%), and infections (9.1\%) on the site of implantation, subcutaneous tunneling or pocket for the pulse generator are the most common complications related to the device. ${ }^{30,31}$ Severe and life-threatening side effects like hemorrhages $(4.5 \%)$ or status epilepticus $(4.5 \%)$ did not occur in our series. ${ }^{30}$

Patients \#3 and \#4 did not have the expected outcome despite an initial good response at 3 months of follow-up. This initial response was likely related to a microlesion effect. This suspected effect was lost at 6 months.

The mechanisms of action of ANT-DBS remain unclear. The ANT is an important center involved in many neuronal networks such as the limbic system (Circuit of Papez) ${ }^{32}$ and the corticothalamo-cortical circuit. Both systems have been demonstrated to participate in disorganized spreading of epileptic activity. ${ }^{33}$

The action of DBS is thought to be in relation to its capacity to induce cellular inhibition or excitation which is reflected either in the increment of the seizure threshold or in the disruption of seizure propagation. ${ }^{34}$ Nevertheless, desynchronization has also been suggested to be one of the main mechanisms of action of ANT-DBS. It has been found by means of stereo electroencephalography that high-frequency stimulation applied to the ANT is capable of desynchronizing basal activity of the ipsilateral hippocampus, as well as suppressing its interictal epileptiform activity and disrupting the connectivity across the cortical areas. ${ }^{35}$

Historically, DBS parameters used for epilepsy have been the same as the ones used for treatment of certain movement disorders. There are not enough data to outline the ideal parameters for epilepsy. ${ }^{36}$ The SANTE trial (first randomized clinical trial for DBS in epilepsy) did not find favorable parameters for frequency, voltage, or pulse width after long-term follow-up. ${ }^{30}$ To this day, stimulation parameters include frequency $>100 \mathrm{~Hz}$; voltage at $1-10 \mathrm{~V}$ for stimulation of ANT. No significant difference between cycling or continuous stimulation was concluded in one open-label study with long-term follow-up. ${ }^{19}$ Another prospective cohort study showed that there is no significant difference between unilateral or bilateral ANT stimulation. ${ }^{37}$

In our series, the setting parameters and the turning ON time differed slightly from the SANTE trial.

For the patients \#1 and \#6, the DBS was turned ON 48 hours and 1 week, respectively, after implantation instead of a month later as SANTE study. The DBS for patients \#2 and \#3 were turned ON 1 month after implantation, similar to SANTE study. For the patients \#4 and \#5, the DBS stimulation was started at 2 months due to their tendency to develop frequent BTC seizures and status epilepticus, and the last one for the patient \#4 who finally died because of SUDEP.

Regarding the DBS parameters, interestingly, there were not significant variations (specialty in voltage) between patients who had good response and those parameters described in the SANTE study. On the other hand, patients who did not benefit from the DBS were stimulated with higher voltages than $6 \mathrm{~V}$, which is keeping with the conclusion of SANTE trial in terms of nonsignificant differences with voltages between 1 and $10 \mathrm{~V} .^{30}$ 
At the end, the clinician who follows the patient will have to optimize stimulation parameters based on the seizure response and feedback from the patient.

In conclusion, DBS of the ANT is an effective and low-risk alternative for patients who have TRE and are not candidates for surgical treatment or have failed previous surgical treatments.

It has been our experience that perhaps those with multifocal and lesional epilepsy (likely regional) would benefit the most. Further investigations with clinical trials are required to figure out optimal parameters of stimulation.

\section{ACKNOWLEDGMenTs}

The authors gratefully acknowledge the support received from Dr. Eduardo Castillo-Leon, Emory University, for the statistical analysis.

\section{Disclosures}

Dr. Burneo reports grants and personal fees from Jack Cowin Endowed Chair in Epilepsy Research (Western University), other from Eisai, other from Sunovion, and outside the submitted work. The remaining authors have nothing to disclose.

\section{Statement of Authorship}

MLH was responsible for the design of the study, collected and analyzed the data, reviewed the literature on the topic, and was in charge of the writing of the manuscript. ASM collected and analyzed the data. AP and KMD provided the detail of surgical interventions. JGB is the corresponding author, was responsible for the idea and helped with the design of the study, provided all the information presented in the study, and interpreted the results. All the authors reviewed and approved the drafts and the final version of the manuscript.

\section{REFERENCES}

1. Kwan P, Brodie MJ. Early identification of refractory epilepsy. N Eng J Med. 2000;342:314-19.

2. Fiest K, Sauro K, Wiebe S, et al. Prevalence and incidence of epilepsy: a systematic review and meta-analysis of international studies. Neurology 2017;88:296-303.

3. Murray C, Vos T, Lozano R, et al. Disability-adjusted life years (DALYs) for 291 diseases and injuries in 21 regions, 1990-2010: a systematic analysis for the Global Burden of Disease Study 2010. Lancet 2012;380:2197-223.

4. Picot M, Baldy-Moulinier M, Daurès J, Dujols P, Crespel A. The prevalence of epilepsy and pharmacoresistant epilepsy in adults: a population-based study in a Western European country. Epilepsia 2008;49:1230-38.

5. Kwan P, Arzimanoglou A, Berg A, et al. Definition of drug resistant epilepsy: consensus Proposal by the ad hoc Task Force of the ILAE Commission on Therapeutic Strategies. Epilepsia 2010. 51:1069-77.

6. Téllez-Zenteno J, Dhar R, Wiebe S. Long-term seizure outcomes following epilepsy surgery: a systematic review and metaanalysis. Brain 2005;128:1188-98.

7. Jobst BC, Cascino GD. Resective epilepsy surgery for drug resistant focal epilepsy: a review. JAMA 2015;313:285-93.

8. West S, Nolan S, Cotton J, et al. Surgery for epilepsy. Cochrane Database Syst Rev 2015;(7):CD010541.

9. Michael Li, Cook M. Deep brain stimulation for drug-resistant epilepsy. Epilepsia. 2017;59:273-90.

10. Möttönen T, Katisko J, Haapasalo $J$, et al. Defining the anterior nucleus of the thalamus (ANT) as a deep brain stimulation target in refractory epilepsy: delineation using $3 \mathrm{~T}$ MRI and intraoperative microelectrode recording. Neuroimage: Clinical. 2015;7:823-29.

11. Scheffer I, Berkovic S, Capovilla G, et al. ILAE classification of the epilepsies: position paper of the ILAE Commission for Classification and Terminology. Epilepsia. 2017;58:512-21.

12. Upton A, Amin I, Garnett S, Springman M, Nahamias C, Cooper I. Evoked metabolic responses in the limbic-striate system produced by stimulation of anterior thalamic nucleus in man. Pacing Clin Electrophysiol. 1987;10:217-25.

13. Andrade DM, Zumsteg D, Hamani C, et al. Long-term follow-up of patients with thalamic deep brain stimulation for epilepsy. Neurology. 2006;66:1571-73.

14. Kerrigan JF, Litt B, Fisher RS, et al. Electrical stimulation of the anterior nucleus of the thalamus for the treatment of intractable epilepsy. Epilepsia. 2004;45:346-54.

15. Lee KJ, Jang KS, Shon YM. Chronic deep brain stimulation of subthalamic and anterior thalamic nuclei for controlling refractory partial epilepsy. Acta Neurochir. Suppl. 2006;99:87-91.

16. Lee KJ, Shon YM, Cho CB. Long-term outcome of anterior thalamic nucleus stimulation for intractable epilepsy. Stereotact Funct Neurosurg. 2012;90:379-85.

17. Yoon-Sang Oh, Kim HJ, Lee KJ, Kim YI, Lim S-C, Shon Y-M. Cognitive improvement after long-term electrical stimulation of bilateral anterior thalamic nucleus in refractory epilepsy patients. Seizure. 2012;21:183-87.

18. Lehtimäki K, Mötönen T, Järventausta $K$, et al. Outcome based definition of the anterior thalamic deep brain stimulation target in refractory epilepsy. Brain Stimul. 2016;9:268-75.

19. Lim S-N, Lee S-T, Tsai Y-T, et al. Electrical stimulation of the anterior nucleus of the thalamus for intractable epilepsy: a longterm follow-up study. Epilepsia. 2007;48:342-47.

20. Osorio I, Overman J, Giftakis J, Wilkinson S. High frequency thalamic stimulation for inoperable mesial temporal epilepsy. Epilepsia. 2007;488:1561-71.

21. Andrade DM, Hamani C, Lozano AM, Wennberg R. Dravet syndrome and deep brain stimulation: seizure control after 10 years of treatment. Epilepsia. 2010;51:1314-16.

22. Van Gompel JJ, Klassen BT, Worrell GA, et al. Anterior nuclear deep brain stimulation guided by concordant hippocampal recording. Neurosurg Focus. 2015;38:E9.

23. Piacentino M, Durisotti C, Garofalo P, et al. Anterior thalamic nucleus deep brain Stimulation (DBS) for drug-resistant complex partial seizures (CPS) with or without generalization: long-term evaluation and predictive outcome. Acta Neurochir (Wien). 2015;157:1525-32.

24. Krishna V, King NKK, Sammartino F, et al. Anterior nucleus deep brain stimulation for refractory epilepsy: insights into patterns of seizure control and efficacious target. Neurosurgery. 2016;78:802-11.

25. Franco A, Pimentel J, Campos A, et al. Stimulation of the bilateral anterior nuclei of the thalamus in the treatment of refractory epilepsy: two cases of subcortical band heterotopia. Epileptic Disord. 2016;18:426-30.

26. Valentín A, Selway RP, Amarouche M, et al. Intracranial stimulation for children with epilepsy. Eur $\mathrm{J}$ Paediatr Neurol. 2017;21:223-31.

27. Nora $T$, Heinonen $H$, Tenhunen $M$, et al. Stimulation induced electrographic seizures in deep brain stimulation of the anterior nucleus of the thalamus do not preclude a subsequent favorable treatment response. Front Neurol. 2018;9:66.

28. Piacentino M, Beggio G, Zordan L, Bonanni P. Hippocampal deep brain stimulation: persistent seizure control after bilateral extracranial electrode fracture. Neurol Sci. 2018;39:1431-35.

29. Jarvenpaa S, Rosti-Otajarvi E, Rainesalo S, Laukkanen L, Lehtimaki K, Peltola J. Executive functions may predict outcome in deep brain stimulation of anterior nucleus of thalamus for treatment of refractory epilepsy. Front Neurol. 2018;9:324.

30. Fisher R, Salanova V, Witt T, et al. Electrical stimulation of the anterior nucleus of thalamus for treatment of refractory epilepsy. Epilepsia. 2010;51:899-908.

31. Salanova V, Witt T, Worth R, et al. Long-term efficacy and safety of thalamic stimulation for drug-resistant partial epilepsy. Neurology. $2015 ; 84: 1017-25$. 
32. Hodaie M, Wennberg R, Dostrovsky J, Lozano A. Chronic anterior thalamus stimulation for intractable epilepsy. Epilepsia. 2002;43:603-608.

33. Kim HY, Hur YJ, Kim HD, Park KM, Kim SE, Hwang TG. Modification of electrophysiological activity pattern after anterior thalamic deep brain stimulation for intractable epilepsy: report of 3 cases. J Neurosurg 2017. 126;2028-35.

34. Wu C, Sharan A. Neurostimulation for the treatment of epilepsy: a review of current surgical interventions. Neuromodulation. 2013;16:10-24.
35. Tao Yu, Xueyuan Wang, Yongjie Li, et al. High-frequency stimulation of anterior nucleus of thalamus desynchronizes epileptic network in humans. Brain. 2018;141:2631-43.

36. Zangiabadi N, Ladino L, Sina F, Orozco-Hernández J, Carter A, Téllez-Zenteno J. Deep brain stimulation and drug-resistant epilepsy: a review of the literature. Front Neurol. 2019;10:601.

37. Cukiert A, Cukiert CM, Burattini JA, Lima AM. Seizure outcome after hippocampal deep brain stimulation in a prospective cohort of patients with refractory temporal lobe epilepsy. Seizure. 2014;23:6-9. 